\title{
Enhancing predictability by increasing nonlinearity in ENSO and Lorenz systems
}

\author{
Z. Ye and W. W. Hsieh \\ Department of Earth and Ocean Sciences, University of British Columbia Vancouver, BC V6T 1Z4, Canada \\ Received: 19 March 2007 - Revised: 30 October 2007 - Accepted: 12 September 2008 - Published: 29 October 2008
}

\begin{abstract}
The presence of nonlinear terms in the governing equations of a dynamical system usually leads to the loss of predictability, e.g. in numerical weather prediction. However, for the El Niño-Southern Oscillation (ENSO) phenomenon, in an intermediate coupled equatorial Pacific model run under the 1961-1975 and the 1981-1995 climatologies, the latter climatology led to longer-period oscillations, thus greater predictability. In the Lorenz (1963) 3-component chaos system, by adjusting the model parameters to increase the nonlinearity of the system, a similar increase in predictability was found. Thus in the ENSO and Lorenz systems, enhanced nonlinearity from changes in the governing equations could produce longer period oscillations with increased predictability.
\end{abstract}

\section{Introduction}

Predicting the future state of the El Niño-Southern Oscillation (ENSO), given its present state, is an important problem in climate research. Interdecadal changes in ENSO predictability are widely noted in different numerical models (Ji et al., 1996; Chen et al., 2004). An (2004) pointed out that the interdecadal change in predictability was related to the interdecadal change in ENSO asymmetry (between the warm El Niño states and the cool La Niña states) and nonlinearity. Changes in the mean climate state could produce changes in ENSO properties, including predictability (Kirtman and Schopf, 1998; An and Wang, 2000; Ye and Hsieh, 2006). Changes in ENSO properties under increased greenhouse gases have been found using datasets from the 4th Assessment Report of Intergovernmental Panel on Climate Change (IPCC-AR4) (Ye and Hsieh, 2008).

Correspondence to: Z. Ye

(zye@ pacific.jpl.nasa.gov)
There is evidence that nonlinear effects play an important role in ENSO properties. Münnich et al. (1991) suggested that nonlinear effects, more specifically a period-doubling bifurcation, led to the 4-yr ENSO period, based on their simple delayed-oscillator model. Nonlinear effects on the ENSO period were also discussed by Eccles and Tziperman (2004), while An and Jin (2004) showed that the nonlinear terms in the heat equation were responsible for the remarkable asymmetry between the warm and cool ENSO states. However, there has been some debate on whether ENSO is primarily a self-sustained nonlinear system (Zebiak and Cane, 1987; Jin et al. 1994) or a damped linear system with stochastic atmospheric forcing (Penland and Sardeshmukh, 1995), i.e. whether the role of nonlinearity in ENSO is primary or secondary. In this paper, we concentrate on the first possibility to explore the nonlinearity and ENSO interdecadal predictability. An intermediate coupled model data based on two different climatological mean states (corresponding to the 1961-1975 and the 1981-1995 climate regimes) were used to analyze the nonlinearity, period and predictability. We also examined the well-known Lorenz nonlinear system (Lorenz, 1963) for comparison. Section 2 contains the results from the coupled model, where the nonlinearity, period and predictability of ENSO based on different climatological mean states are shown. Section 3 presents the results from the Lorenz system.

\section{Intermediate coupled model results}

The modified Zebiak and Cane (1987) coupled model (see Ye and Hsieh, 2006) was used to simulate ENSO for 2300 years, with the last 2100 years of data analyzed. In this model, the background seasonal climatologies (sea surface temperature (SST), oceanic surface layer currents and associated upwelling/downwelling, surface wind stress (WS) and surface wind divergence) were prescribed with the data

Published by Copernicus Publications on behalf of the European Geosciences Union and the American Geophysical Union. 
from the 1961-1975 regime and the 1981-1995 regime in two separate model runs. A 3-month running mean was applied to the coupled model data. The 2100-year data record was divided into 21 equal segments, allowing us to compute ensemble means over 21 members, instead of the single runs reported by Ye and Hsieh (2006).

One way to characterize the asymmetry between El Niño and La Niña is by nonlinear principal component analysis (NLPCA) (which fits a curve to account for the maximum variance in the dataset, instead of a straight line as in classical PCA) (Hsieh, 2004). The spatial patterns from the first NLPCA mode averaged over 21 ensemble members for strong El Niño and La Niña are shown in Fig. 1. For strong El Niño the center of the SST anomalies (defined by the maximum value of the anomalies, as marked by the " $\mathrm{H}$ " in Fig. 1c) under the 1981-1995 climatology had an eastward displacement of about $11^{\circ}$ relative to that under the 1961-1975 climatology (as marked by the "H" in Fig. 1a). The asymmetry change can be seen more clearly in the SST anomalies along equator averaged between $5^{\circ} \mathrm{S}$ and $5^{\circ} \mathrm{N}$ (Fig. 1e and f). For La Niña, although the low "L" was not shifted (Fig. 1b and d), there was a westward displacement of cool anomalies in the later regime (Fig. 1f). The asymmetry between El Niño and La Niña, hence the nonlinearity, increased under the 1981-1995 climatology.

The histogram of the first principal component (PC) time series for all ensemble members are shown in Fig. 2. The frequency of occurrence for the strong El Niño (corresponding to large positive PC1) is higher under the 1981-1995 climatology, while the occurrence frequency for the strong La Niña (corresponding to large negative PC1) is lower under the 1981-1995 climatology. A similar conclusion can be obtained from the Niño 3 index results (averaged SST anomalies in $90^{\circ}-150^{\circ} \mathrm{W}, 5^{\circ} \mathrm{S}-5^{\circ} \mathrm{N}$ ). The number of times when the Niño 3 indices are greater than $3^{\circ} \mathrm{C}$ are 163 and 238 months for the pre-shift and post-shift regimes, respectively.

One way to characterize the nonlinearity in the data is to compare the percentage variance explained by the first mode from PCA with that from NLPCA (Hsieh, 2004). Let

$\delta=\left(P_{N L}-P_{L}\right) / P_{L}$,

where $P_{N L}$ is the percentage variance explained by the NLPCA mode 1 , and $P_{L}$, by the (linear) PCA mode 1 (Ye and Hsieh, 2006).

The ensemble mean \pm 1 standard error for $\delta$ is $8.1 \% \pm 0.1 \%$ for the simulation under the 1961-1975 climatology and $8.8 \% \pm 0.1 \%$ under the 1981-1995 climatology, indicating enhanced nonlinearity under the latter climatology. This enhanced nonlinearity arose from the greater asymmetry found in the SST anomaly patterns between El Niño and La Niña under the 1981-1995 climatology (Ye and Hsieh, 2006). The asymmetry in turn is produced by the nonlinear terms in the heat budget of the upper ocean (An and Jin, 2004). Ye and
Hsieh (2006) showed that in this simple model the change in ENSO properties was mainly due to changes in the climatology of the surface wind and surface wind divergence than to changes in the climatology of the upper ocean.

An alternative, dynamical measure of the nonlinearity is the size of the nonlinear terms relative to the size of the linear terms in the ocean surface-layer temperature equation in the ENSO coupled model. From Ye and Hsieh (2006), the equation is

$$
\begin{aligned}
& \frac{\partial T}{\partial t}=-\boldsymbol{u}_{1} \cdot \nabla(\bar{T}+T)-\overline{\boldsymbol{u}}_{1} \cdot \nabla T-\left\{M\left(\bar{w}_{s}+w_{s}\right)-M\left(\bar{w}_{s}\right)\right\} \\
& \times \frac{\partial \bar{T}}{\partial z}-M\left(\bar{w}_{s}+w_{s}\right) \frac{T-T_{e}}{H_{1}}-\alpha_{s} T-K_{t} \frac{T-T_{e}}{H_{1}}+A_{h} \triangle_{h} T,
\end{aligned}
$$

where the last two terms are used to simulate vertical mixing and horizontal diffusion, respectively. Here $\overline{\boldsymbol{u}}_{1}(x, y, t)$ and $\bar{w}_{s}(x, y, t)$ are the prescribed climatological monthly mean horizontal current and upwelling in the surface layer respectively, $\bar{T}(x, y, t)$ is the prescribed mean SST, $\partial \bar{T}(x) / \partial z$ the prescribed mean vertical temperature gradient, the mean surface layer depth $H_{1}=50 \mathrm{~m}$, the diffusion coefficient $\alpha_{s}=(125 \text { day })^{-1}, K_{t}=2.5 \times 10^{-5} \mathrm{~m} \mathrm{~s}^{-1}, A_{h}=2000 \mathrm{~m}^{2} \mathrm{~s}^{-1}$, the function $M$ is defined by

$M(x)= \begin{cases}0, & x \leq 0 \\ x, & x>0\end{cases}$

and the entrainment velocity is

$w_{s}=H_{1}\left(\frac{\partial u_{1}}{\partial x}+\frac{\partial v_{1}}{\partial y}\right)$.

The entrainment temperature anomaly, $T_{e}$, is given by

$T_{e}=\gamma T_{\mathrm{sub}}+(1-\gamma) T$,

where $\gamma=0.75$, and $T_{\text {sub }}$ is calculated from the model upperlayer depth anomaly $h$ by a nonlinear empirical parameterization scheme using a neural network model (Ye and Hsieh, 2006). We then compare the nonlinear terms relative to the linear terms in Eq. (2) by computing the ratio

$$
\begin{aligned}
& \beta_{\mathrm{SST}}=\frac{\left\langle\left|\boldsymbol{u}_{1} \cdot \nabla T\right|+\left|\left\{M\left(\bar{w}_{s}+w_{s}\right)-M\left(\bar{w}_{s}\right)\right\} \times \frac{\partial \bar{T}}{\partial z}\right|+\right.}{\left\langle\left|\boldsymbol{u}_{1} \cdot \nabla \bar{T}\right|+\left|\overline{\boldsymbol{u}}_{1} \cdot \nabla T\right|+\left|\alpha_{s} T\right|+\right.} \\
& \frac{\left.\left|M\left(\bar{w}_{s}+w_{s}\right) \frac{T-T_{e}}{H_{1}}\right|+\left|K_{t} \frac{T_{e}}{H_{1}}\right|\right\rangle}{\left.\left|K_{t} \frac{T}{H_{1}}\right|+\left|A_{h} \triangle_{h} T\right|\right\rangle}
\end{aligned}
$$

where $\langle\ldots\rangle$ denotes the temporal mean. The average $\beta_{\mathrm{SST}}$ in the Niño 3.4 region $\left(120^{\circ} \mathrm{W}-170^{\circ} \mathrm{W}, 5^{\circ} \mathrm{S}-5^{\circ} \mathrm{N}\right)$ is 0.77 for the simulation under the 1961-75 climatology and 0.85 under the 1981-1995 climatology, while over the whole tropical Pacific, $\beta_{\text {SST }}$ is 0.72 (earlier climatology) versus 0.75 (latter climatology). Thus $\beta_{\mathrm{SST}}$ also indicates enhanced nonlinearity under the latter climatology. 
(a) SST, Pre-shift regime (El Nino)

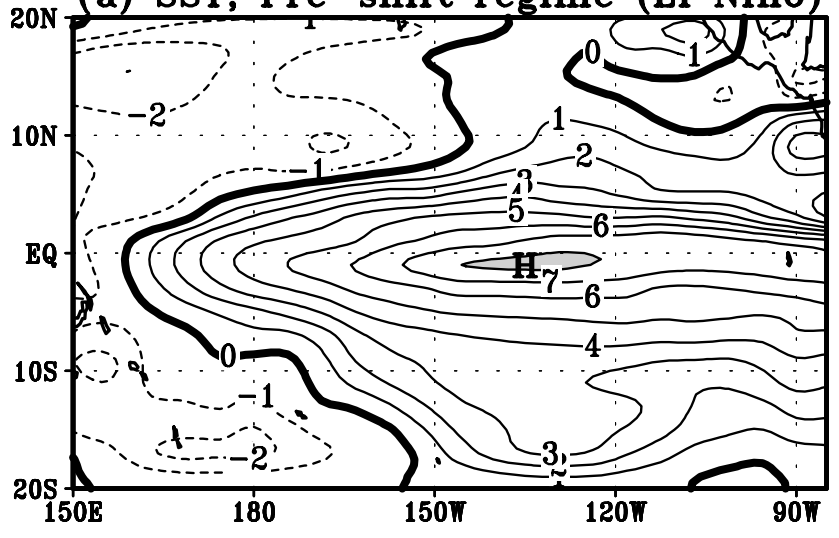

(c) SST, Post-shift regime (El Nino)

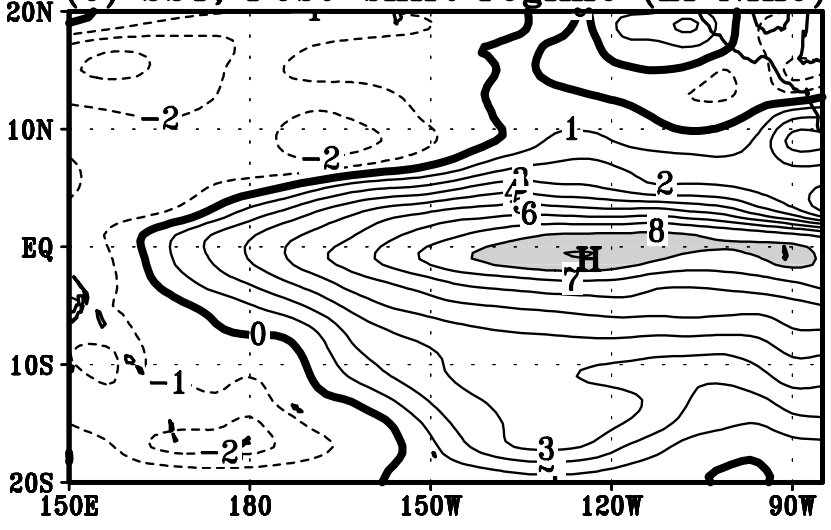

(e) El Nino

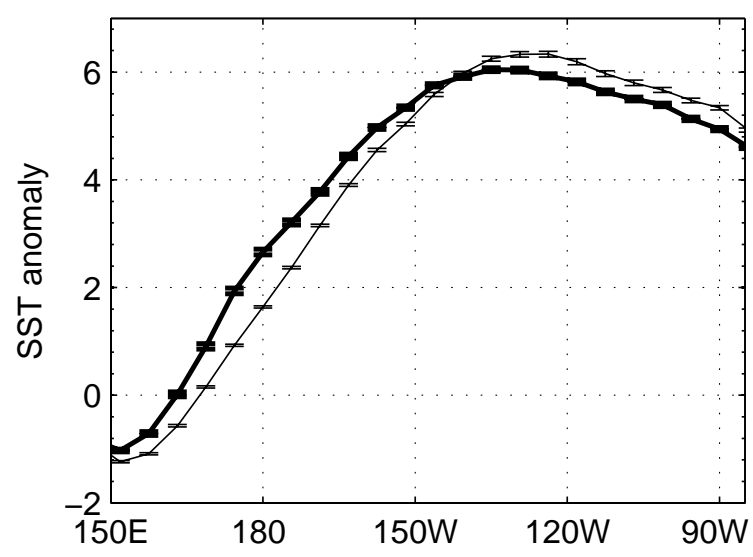

(b) SST, Pre-shift regime (La Nina)

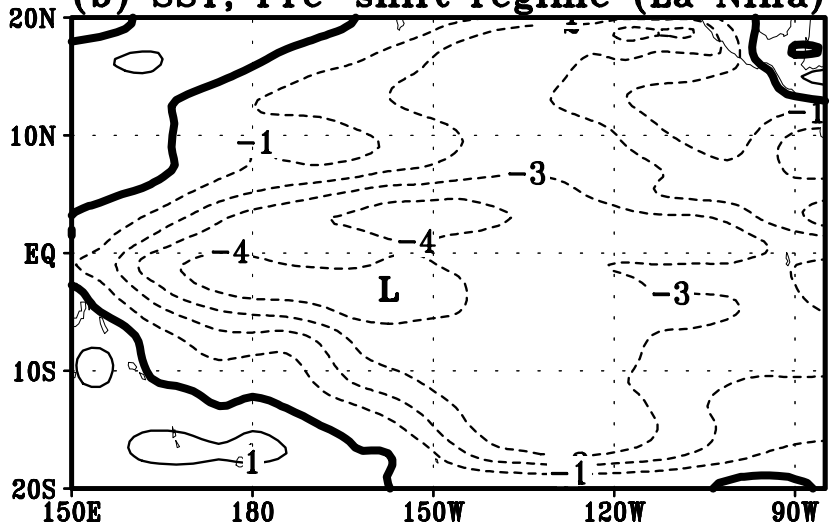

(d) SST, Post-shift regime (La Nina)

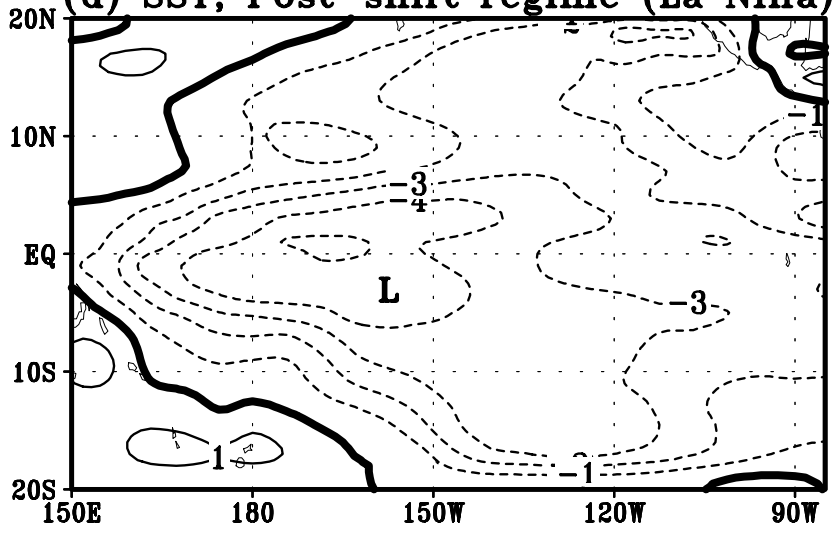

(f) La Nina

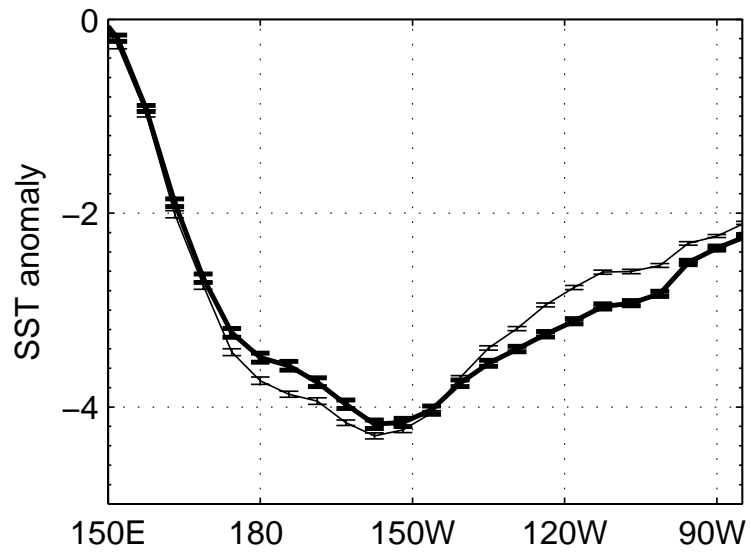

Fig. 1. The SST anomalies $\left({ }^{\circ} \mathrm{C}\right.$ ) from the leading NLPCA mode when the NLPC takes its (a) maximum value (strong El Niño) and (b) minimum value (strong La Niña), as averaged over 21 ensemble members in the experiments using the climatology of the oceanic and the atmospheric fields for the 1961-1975 regime; and similarly for the 1981-1995 regime in (c) and (d), respectively. "L" and "H" mark the location of the lowest and highest values, respectively, and areas $\geq 8^{\circ} \mathrm{C}$ are shaded in (a) and (c). (e) is the average SST anomalies between $5^{\circ} \mathrm{S}$ and $5^{\circ} \mathrm{N}$ of panels (a) and (c), and (f) is the average SST anomalies of (b) and (d), where the thick lines are for the 1961-1975 regime and thin lines for the 1981-1995 regime, with error bars showing \pm 1 standard error. 


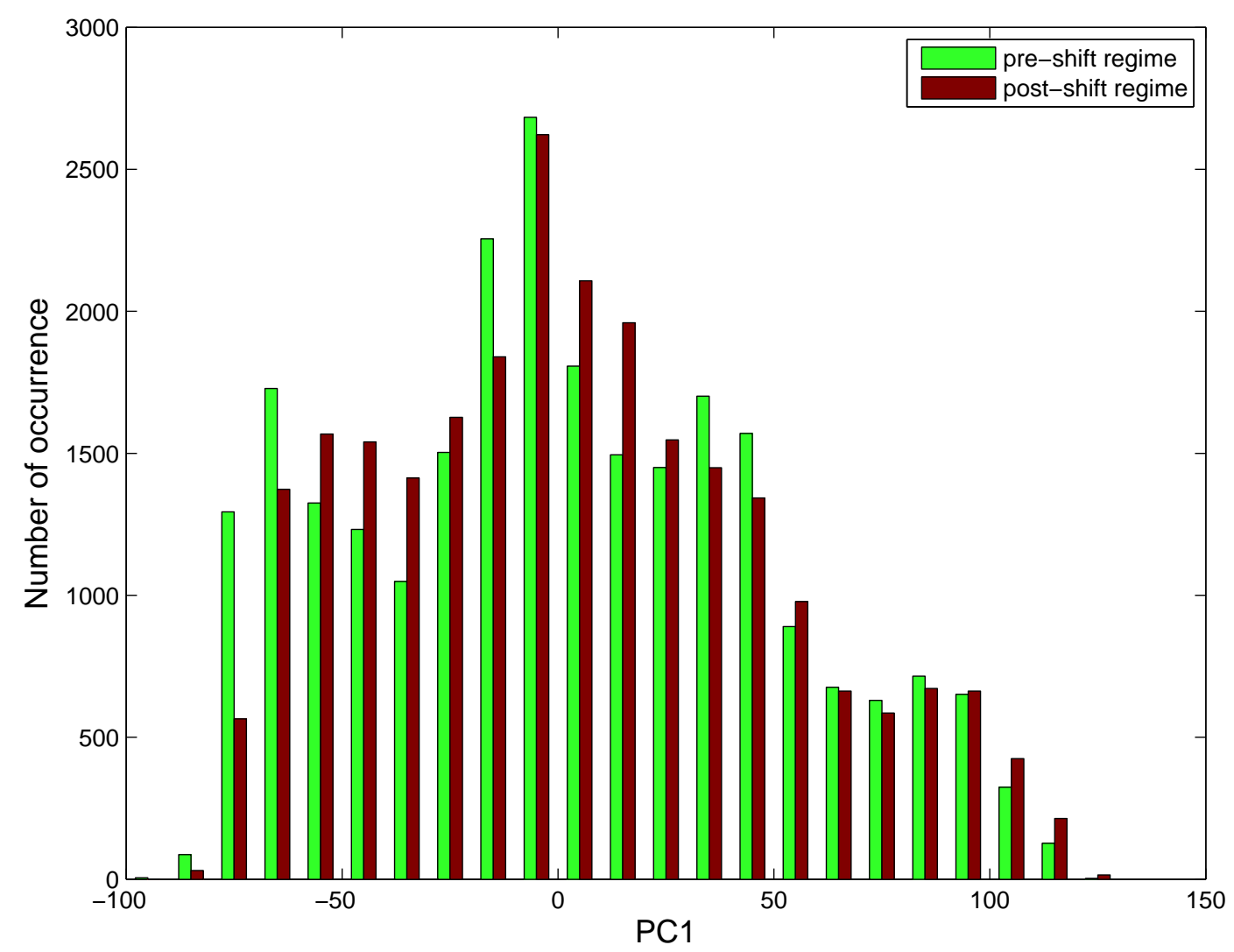

Fig. 2. Histogram of the first PCs for all 21 members for pre-shift climate state run and for post-shift climate state run.

Multi-layer perceptron neural network (NN) models have become popular for performing nonlinear regression, as these models are capable of representing any nonlinear functional relation $\boldsymbol{y}=\boldsymbol{f}(\boldsymbol{x})$ to arbitrary accuracy with generally far fewer parameters than polynomials (Bishop, 1995). The average SST anomaly in the Niño 3.4 region was predicted at lead times from 0 to 15 months by nonlinear regression using NN models with Bayesian regularization as provided by the MATLAB neural network toolbox. At time $t$, the 2 leading principal components from a combined PCA of the normalized SST, zonal and meridional WS anomalies were used as predictors to forecast the Niño 3.4 SST anomaly at $t+$ lead time. The ensemble-averaged crossvalidated correlation coefficients and mean squared error (MSE) between the predicted and actual Niño 3.4 indices from our coupled model can be used to characterize ENSO predictability (Fig. 3), where enhanced predictability under the 1981-1995 climatology can be seen when the lead time exceeds 3 months. For comparison, the predictability based on linear regression (as used by Ye and Hsieh, 2006) is lower than that based on nonlinear regression (Fig. 3), but gives the same conclusion, i.e. using the 1981-1995 climatology in the coupled model enhanced the ENSO predictability.
Fourier spectral analysis performed on the Niño 3.4 indices from the 2 coupled model runs revealed that the main spectral peak shifted from a period of 49 months under the 1961-1975 regime climatology to 52 months under the 1981-1995 regime climatology (Fig. 4). The lead times for attaining a correlation skill of 0.95 by nonlinear regression (Fig. 3a) are 3.4 and 3.8 months for the pre- and post1980 climatologies, respectively. Dividing these predictability lead times by the respective spectral periods of 49 and 52 months for the two regimes yielded a lead time equal to 0.069 and 0.073 cycle for two regimes, respectively. In other words, when predicting 0.07 cycle ahead, the nonlinear regression method can attain a correlation skill of 0.95 in this noiseless coupled model of ENSO under both the pre- and post-1980 climatologies.

Chen et al. (2004) argued that an increased ENSO amplitude in the post-1980 regime led to increased predictability. In our noiseless model, an increased in amplitude does not improve the signal-to-noise ratio (hence the predictability). Although the increase in nonlinearity can be associated with increase in both the amplitude and period, in a noiseless ENSO system, the increased in predictability comes from the increased period only, whereas in a noisy system, increased predictability comes from both the increase in period and amplitude. 
(a)

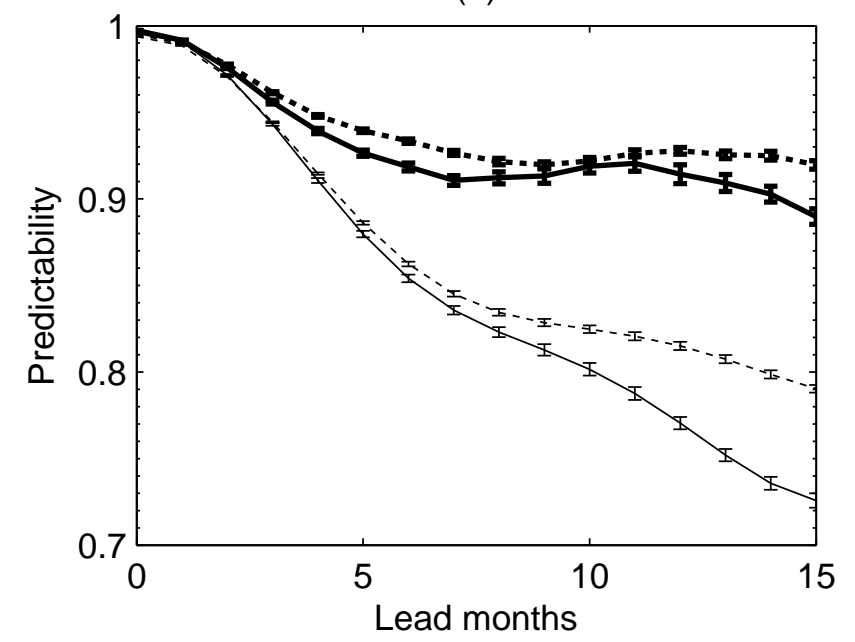

(b)

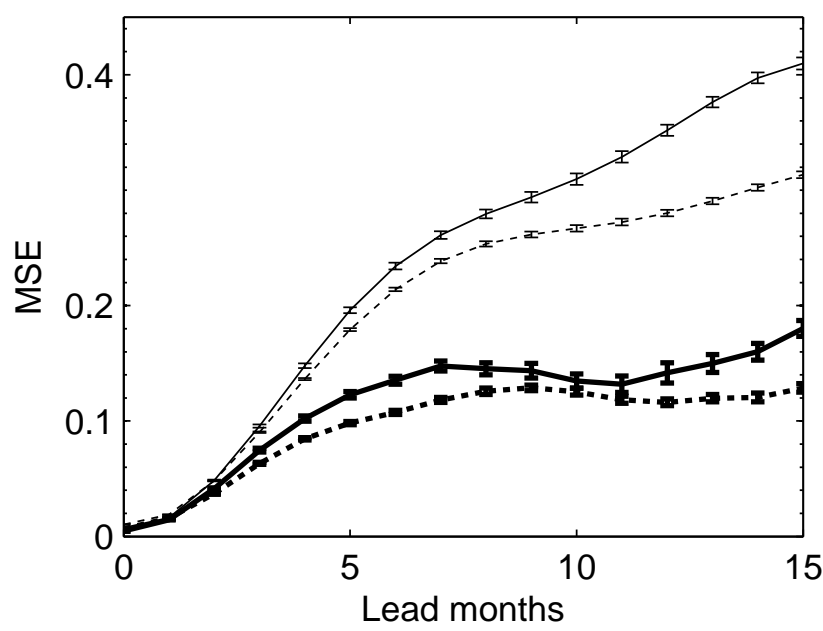

Fig. 3. Ensemble mean predictability and MSE of the Niño 3.4 SST anomaly index, as given by the cross-validated (a) correlation and (b) MSE between the predicted and actual index in the coupled model using climatologies from the 1961-1975 regime (solid lines) and the 1981-1995 regime (dashed lines). The thick lines are from nonlinear regression, the thin lines, linear regression. Crossvalidation was performed by dividing each 100-year data record into five segments, where for each segment chosen to test the forecast correlation skills, the other four were used to build the forecast model. Error bars indicate \pm 1 standard error of the ensemble mean.

In the stability analysis of Federov and Philander (2001), increasing the mean surface temperature amounts to increasing their model parameter $\Delta T$ (the mean temperature difference across the thermocline), which from the increased stability, leads to slower growth of instabilities hence longer ENSO period as seen in their Fig. 12a. This change in period is not a linear effect - when we turn off all the nonlinear terms in the surface temperature Eq. (2) of our coupled

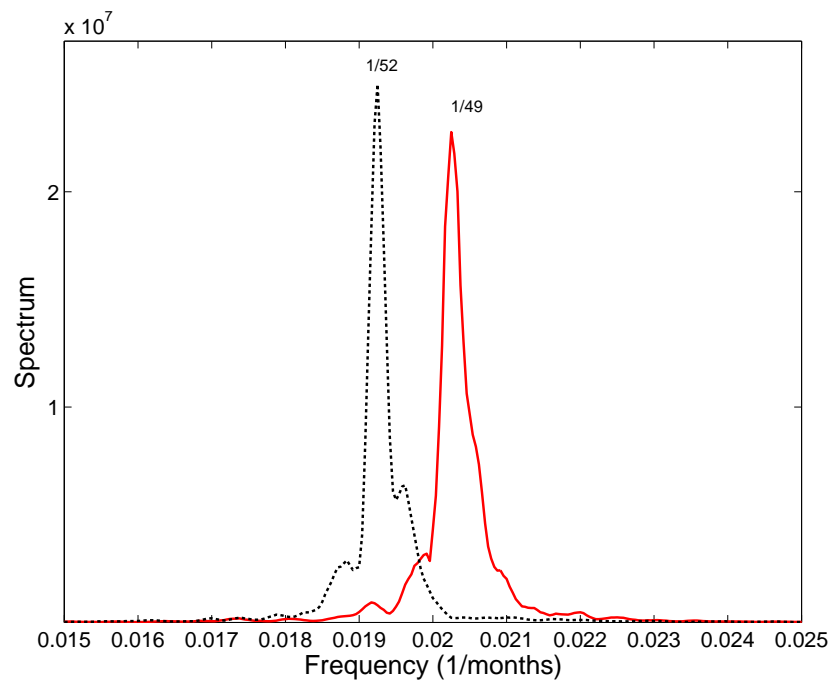

Fig. 4. Spectrum of the Niño 3.4 SST anomaly index in the coupled model using climatologies from the pre-shift regime (solid curve) and post-shift regime (dashed curve).

model, the SST anomalies display no longer predominantly interannual variability at the 4-5 year period, but only variability mainly around the annual period for both the pre- and post-1980 climatologies. Thus the change in the climatology, with warmer SST in the post-1980 regime (Ye and Hsieh, 2006, Fig. 3), would induce stronger nonlinearity and longer ENSO period, thereby enhancing the predictability.

There is a debate regarding the processes that limit the predictability of ENSO (Kirtman, 1998). Understanding how intraseasonal variations in the tropical Pacific affect ENSO prediction and predictability is complicated by the fact that there is no clear understanding of the mechanisms that lead to its irregularity and ultimately the loss of predictability. It was argued that intraseasonal variability acts as a fundamental limit to ENSO predictability (Kleeman and Moore, 1997). However, at least for the Zebiak-Cane coupled model, the effects of the intraseasonal forcing generally play a minor role to ENSO (Zebiak, 1989). Since the experiments are noise free in this paper, intraseasonal variability is not an essential component of ENSO.

\section{Lorenz attractor data}

We next turn to a very different nonlinear system to see if the same behavior can be found. The Lorenz (1963) nonlinear system is given by

$d x / d t=-a x+a y$,
$d y / d t=-x z+b x-y$,
$d z / d t=x y-c z$, 
Table 1. Ensemble mean results for Lorenz systems with different parameter pairs.

\begin{tabular}{lcccc}
\hline Case & $\begin{array}{c}1 \\
\text { (Lorenz, 1963) }\end{array}$ & 2 & $\begin{array}{c}3 \\
(\text { Basu, 2002) }\end{array}$ & $\begin{array}{c}4 \\
\text { (Elsner and Tsonis, 1992) }\end{array}$ \\
\hline param $(a, b, c)$ & $(10,28,8 / 3)$ & $(13,36.4,3.47)$ & $(16,45.92,4.0)$ & $(16,120.1,4.0)$ \\
nonlinearity $\beta_{y}$ & 0.033 & 0.026 & 0.020 & 0.008 \\
nonlinearity $\beta_{z}$ & 0.400 & 0.307 & 0.271 & 0.260 \\
x period (step) & 15.2 & 12.4 & 11.2 & 10.4 \\
z period (step) & 15.2 & 11.3 & 9.2 & 5.4 \\
\hline
\end{tabular}

where $x, y, z$ are proportional to the intensity of convective motion, the temperature gradient in the horizontal and vertical directions, respectively. A fourth-order RungeKutta method was used to integrate the equations (from $t=-15$ to 60 at time steps of 0.05$)$ from initial conditions $(x, y, z)=(-9.42,-9.43,28.3)$, with parameters $a=10$, $b=28$, and $c=8 / 3$. Data from $t=0$ to 60 were analyzed.

The degree of nonlinearity of the system can be characterized by 2 parameters:

$\beta_{y}=\frac{\langle|x z|\rangle}{\langle|b x|+|y|\rangle}, \quad \beta_{z}=\frac{\langle|x y|\rangle}{\langle|c z|\rangle}$,

where $\langle\ldots\rangle$ denotes the temporal mean, $\beta_{y}$ measures the size of the nonlinear term relative to the size of the linear terms in Eq. (8), and similarly, $\beta_{z}$ for Eq. (9).

We next perform four numerical experiments by varying the parameters $(a, b, c)$ : Case 1 uses the same parameters as in Lorenz (1963) (Table 1); Case 2 multiplies the parameters of Case 1 by the uniform factor of 1.3; Case 3 uses the parameters from Basu and FoufoulaGeorgiou (2002); and Case 4, from Elsner and Tsonis (1992). The nature of the oscillations varies among the four cases (Fig. 5). For each case, 21 ensemble runs were made by adding one percent stochastic noise onto the initial data (i.e., the initial data $x_{t o}, y_{t o}, z_{\text {to }}$ were changed to $\left(1+0.01 \alpha_{1}\right) x_{t o},\left(1+0.01 \alpha_{2}\right) y_{t o},\left(1+0.01 \alpha_{3}\right) z_{t o}$, where $\alpha_{1}$, $\alpha_{2}$ and $\alpha_{3}$ were Gaussian random numbers with zero mean and unit standard deviation).

Table 1 lists $\beta_{y}$ and $\beta_{z}$ calculated from Eq. (10) for the four cases. The main spectral periods $T_{x}$ and $T_{z}$ determined from the power spectra of $x$ and $z$, respectively (Fig. 6) are also listed in Table 1. The spectral behavior of $y$ (not shown) is basically the same as as that for $x$, but $z$ shows sharper spectral peaks than $x$ (Fig. 6). Proceeding inversely from Case 4 to Case 1 , one finds a progressive increase in $\beta_{y}$ and $\beta_{z}$, and in $T_{x}$ and $T_{z}$, indicating that an increase in the nonlinearity of the system coincides with an increase in the spectral period of the oscillations.
Again $(x, y, z)$ at time $t$ were used as predictors in nonlinear regression models to separately predict these three variables at time $t+$ lead time (where the lead time ranged from 0 to 20 time steps). Fig. 7 shows enhanced predictability (with higher correlation coefficient and lower MSE) for $z$ as one proceeds inversely from Case 4 to Case 1, where the nonlinearity of the system increased. This behavior in predictability was also found for $x$ (not shown), though for a given level of correlation skill, $x$ tends to have longer lead times than $z$.

Since relative to $x$, the predictability lead time for $z$ tends to be shorter, and its main spectral period $T_{z}$ is also $\leq T_{x}$ and more sensitive to changes in the Lorenz system parameters (Table 1), we now focus on $z$. Its predictability lead time (for 0.80 correlation skill) divided by the main spectral period $T_{z}$ is $0.69,0.69,0.70$ and 0.65 for Cases 1 to 4 , respectively, i.e. in all 4 cases, the nonlinear regression model when predicting ahead by 0.7 times the period in $z$ can attain a correlation skill of 0.8 for all four cases, thereby demonstrating that the enhanced predictability was basically due to the lengthened period of the oscillations when the nonlinearity of the Lorenz system was enhanced from Case 4 to Case 1 . For the correlation skill of 0.95 , its predictability lead time divided by the main spectral period $T_{z}$ is $0.51,0.50,0.50$ and 0.46 for Cases 1 to 4 , respectively. So a similar conclusion can be drawn regardless of the correlation skill level chosen.

One potential problem with our calculation of $\beta_{y}$ and $\beta_{z}$ is that $(0,0,0)$ has been used as the reference point, whereas the Lorenz attractor has two other equilibrium points (Drazin, 1992) which could also be chosen as the reference point $\left(x_{0}, y_{0}, z_{0}\right)$. If we replace $(x, y, z)$ by $\left(x_{0}+x^{\prime}, y_{0}+y^{\prime}, z_{0}+z^{\prime}\right)$ in Eqs. (7)-(9), then drop the primes for brevity, we get the Lorenz system with respect to the reference point $\left(x_{0}, y_{0}, z_{0}\right)$ :

$$
\begin{aligned}
& d x / d t=-a\left(x+x_{0}\right)+a\left(y+y_{0}\right), \\
& d y / d t=-\left(x+x_{0}\right)\left(z+z_{0}\right)+b\left(x+x_{0}\right)-\left(y+y_{0}\right), \\
& d z / d t=\left(x+x_{0}\right)\left(y+y_{0}\right)-c\left(z+z_{0}\right) .
\end{aligned}
$$



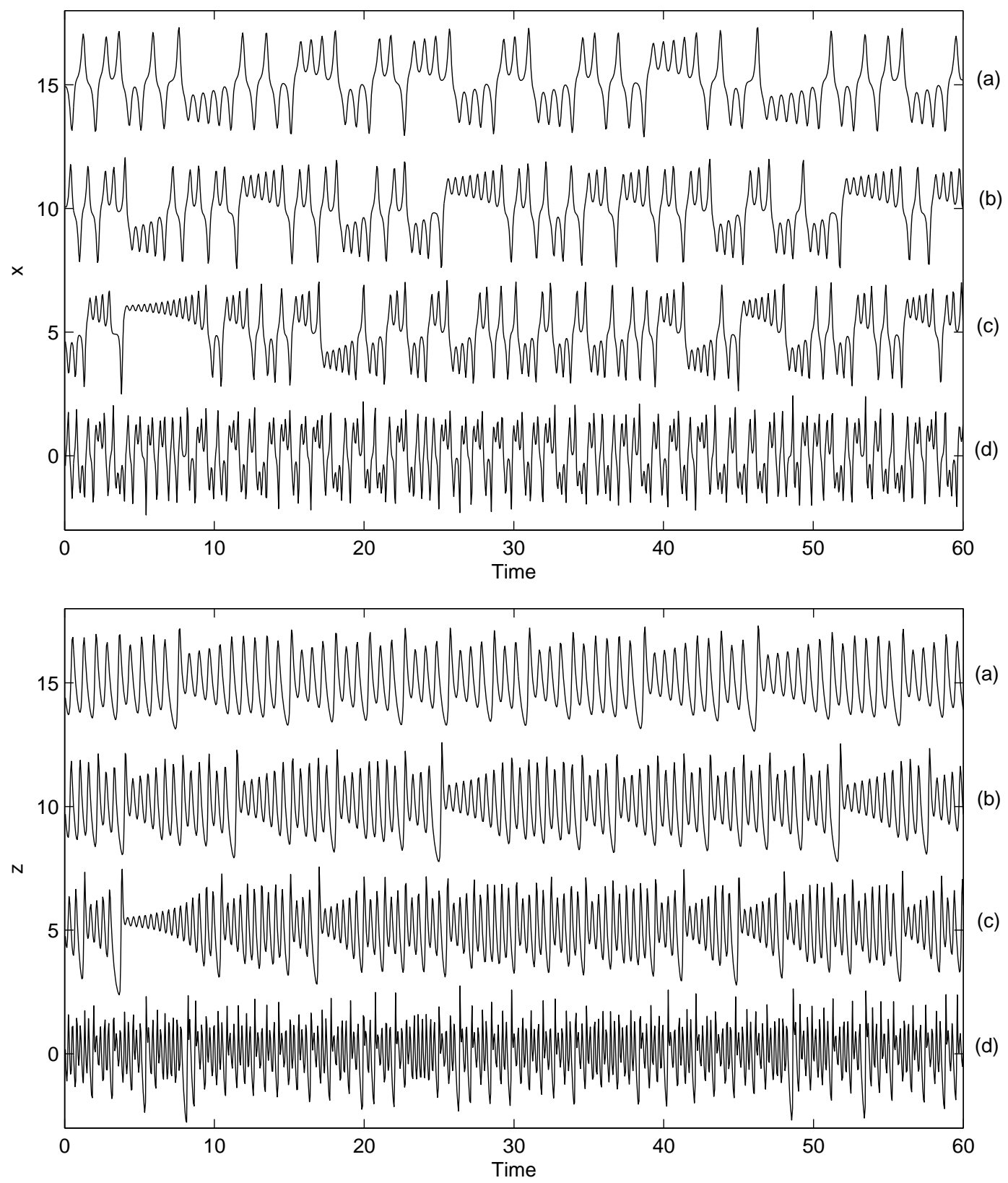

Fig. 5. Normalized time series of $x$ (top panel) and $z$ (bottom panel) in the Lorenz model for (a) Case 1, (b) Case 2, (c) Case 3 and (d) Case 4 , where the time series for different cases are vertically shifted by multiples of 5 for legibility.

The ratios of nonlinear to linear terms in Eqs. (12) and (13) are

$\beta_{y}=\frac{\langle|x z|\rangle}{\left\langle\left|z_{0} x\right|+\left|x_{0} z\right|+|b x|+|y|\right\rangle}, \beta_{z}=\frac{\langle|x y|\rangle}{\left\langle\left|y_{0} x\right|+\left|x_{0} y\right|+|c z|\right\rangle}$

When the reference point is the equilibrium point $(\sqrt{c(b-1)}$, $\sqrt{c(b-1)}, b-1)$, the parameter $\beta_{y}$ is found to be 0.037 , $0.027,0.020,0.008$ for Cases 1 to 4 , respectively; while $\beta_{z}$ is $0.320,0.296,0.260,0.245$ for Cases 1 to 4 . Similar results are obtained if we choose the other equilibrium point $(-\sqrt{c(b-1)},-\sqrt{c(b-1)}, b-1)$ as the reference. Therefore both $\beta_{y}$ and $\beta_{z}$ decrease as we proceed from Case 1 to Case 4, regardless of whether the origin or one of the other 2 equilibrium points has been chosen as the reference point.

A caveat on the Lorenz system: The system is chaotic only over a range of parameter values. Our numerical runs were done over a range where the Lorenz system was chaotic. If the parameters were increased or decreased by the right amount, the Lorenz system could simply converge from the initial state to a single point in the $(x, y, z)$ space. 

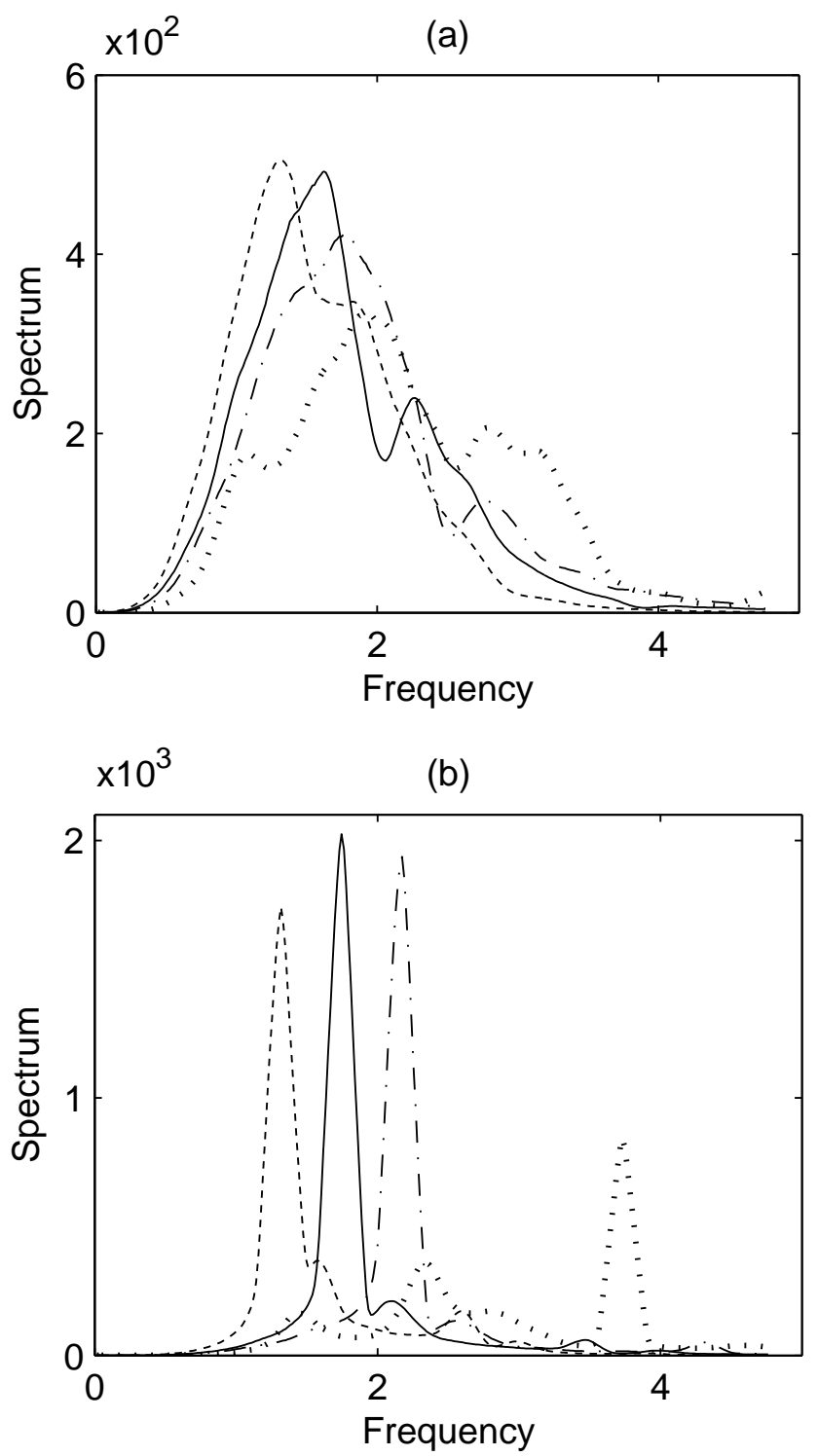

Fig. 6. Ensemble mean power spectrum of the normalized (a) $\mathrm{x}$ and (b) z components. Dash, solid, dash-dot and dotted lines represent Case 1, 2, 3 and 4, respectively.

\section{Summary and conclusion}

Numerical coupled model results show that the predictability of ENSO is closely related to its nonlinearity and period. Under the post-1980s climatology, ENSOs have stronger nonlinearity and longer period. The longer period enhances the system's persistence, leading to better predictability. This behavior was also found in the Lorenz chaotic system, suggesting that it is not unusual for the increased nonlinearity of a climate system to enhance its predictability.
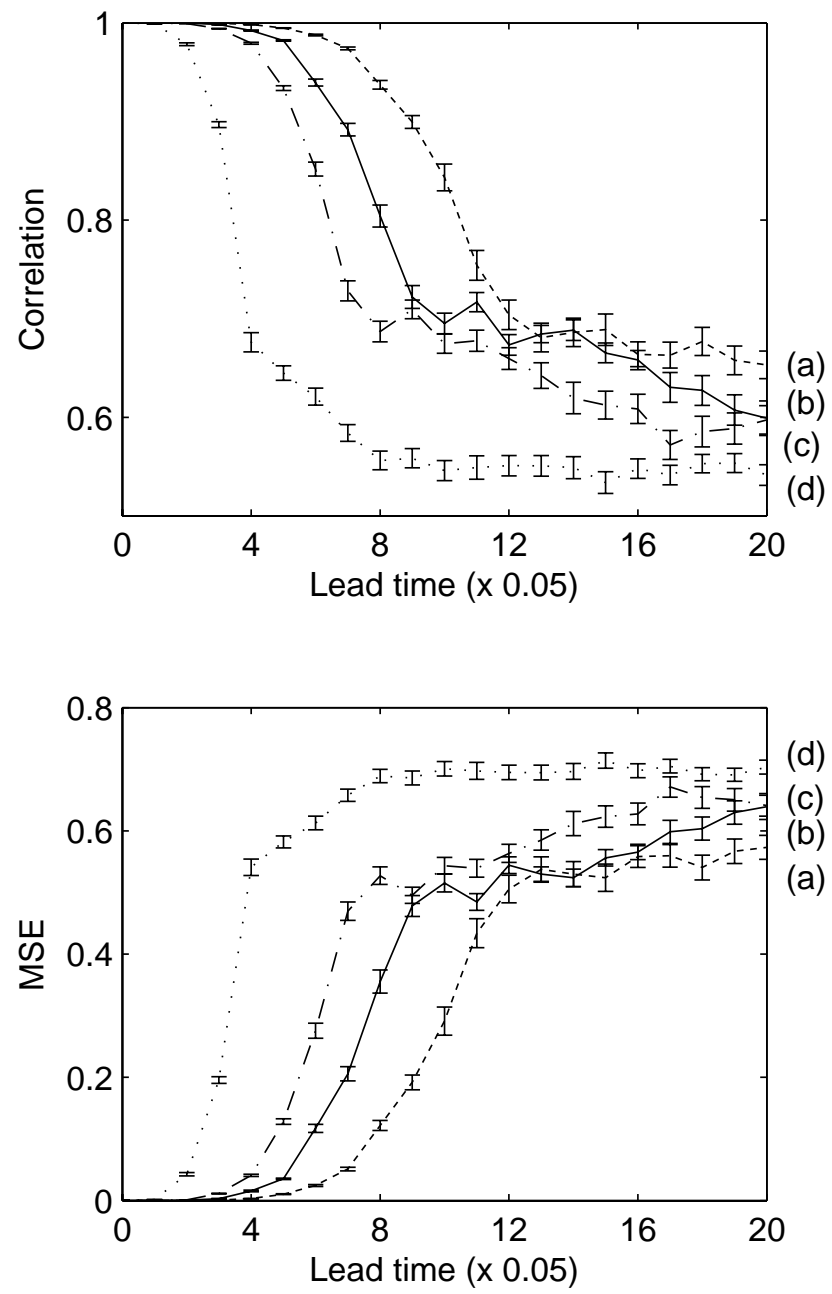

Fig. 7. Ensemble mean predictability in terms of correlation skill (top) and MSE (bottom) of $z$ in the Lorenz system, as given by the cross-validated correlation and MSE between the predicted and actual time series. Dash, solid, dash-dot and dotted lines represent Case 1 (a), 2 (b), 3 (c) and 4 (d), respectively, with error bars showing \pm 1 standard error.

In the ENSO coupled model, when the nonlinear terms in the surface temperature equation are switched off, the SST anomalies display no longer predominantly interannual variability at the $4-5$ year period, but only variability mainly around the annual period for both the pre- and post-1980 climatologies. Similarly, when the nonlinear terms in the Lorenz system are discarded, the system does not oscillate at all. Hence the nonlinear terms in both the ENSO and Lorenz systems play a crucial role in influencing the period of the oscillations hence the predictability. 
Acknowledgements. This work was supported by the Natural Sciences and Engineering Research Council of Canada via Discovery and Strategic grants to W. Hsieh.

Edited by: O. Talagrand

Reviewed by: four anonymous referees

\section{References}

An, S.-I. and Wang, B.: Interdecadal change of the structure of the ENSO mode and its impact on the ENSO frequency, J. Climate, 13, 2044-2055, 2000.

An, S.-I.: Interdecadal changes in the El Niño La-Niña asymmetry, Geophys. Res. Lett., 31, L23210, doi:10.1029/2004GL021699, 2004.

An, S.-I. and Jin, F.-F.: Nonlinearity and asymmetry of ENSO, J. Climate, 17, 2399-2412, 2004.

Basu, S. and Foufoula-Georgiou, E.: Detection of nonlinearity and chaoticity in time series using the transportation distance function, Phys. Lett. A, 301, 413-423, 2002.

Bishop, C. M.: Neural Networks for Pattern Recognition, Clarendon Press, 482 pp., 1995.

Chen, D., Cane, M. A., Kaplan, A., Zebiak, S. E., and Huang, D.: Predictability of El Niño in the past 148 years, Nature, 428, 733 736, 2004.

Drazin, P. G.: Nonlinear Systems, Cambridge University Press, 317 pp., 1992.

Elsner, J. B. and Tsonis, A. A.: Nonlinear prediction, chaos and noise, B. Am. Meteorol. Soc., 73, 49-60, 1992.

Eccles, F. and Tziperman, E.: Nonlinear effects on ENSO's period, J. Atmos. Sci., 61, 474-482, 2004.
Federov, A. V. and Philander, S. G.: A stability analysis of tropical ocean-atmosphere interactions: Bridging measurements and theory for El Niño, J. Clim., 14, 3086-3101, 2001.

Hsieh, W. W.: Nonlinear multivariate and time series analysis by neural network methods, Rev. Geophys., 42, RG1003, doi:10.1029/2002RG000112, 2004.

Ji, M., Leetmaa, A., and Kousky, V. E.: Coupled model predictions of ENSO during the 1980s and the 1990s at the National Centers for Environmental Prediction, J. Climate, 9, 3105-3120, 1996.

Jin, F.-F., Neelin, D., and Ghil, M.: ENSO on the devil's staircase, Science, 264, 70-72, 1994.

Kirtman, B. P. and Schopf, P. S.: Decadal variability in ENSO predictability and prediction, J. Climate, 11, 2804-2822, 1998.

Kleeman, R. and Moore, A. M.: A theory for the limitation of ENSO predictability due to stochastic atmospheric transients, J. Atmos. Sci., 54, 753-767, 1997.

Lorenz, E. N.: Deterministic non-periodic flow, J. Atmos. Sci., 20, 130-141, 1963.

Münnich, M., Cane, M. A., and Zebiak, S. E.: A study of selfexcited oscillations of the tropical ocean-atmosphere system. Part II: Nonlinear cases, J. Atmos. Sci., 48, 1238-1248, 1991.

Penland, C. and Sardeshmukh, P. D.:The optimal-growth of tropical sea-surface temperature anomalies, J. Climate, 8, 1999-2024, 1995.

Ye, Z. and Hsieh, W. W.: The influence of climate regime shift on ENSO, Clim. Dynam., 26, 823-833, doi:10.1007/s00382-0050105-5, 2006.

Ye, Z. and Hsieh, W. W.: Changes in ENSO and associated overturning circulations from enhanced greenhouse gases by the end of the 20th century, J. Climate, 21, 5745-5763, doi:10.11752008JCLI1580.1, 2008.

Zebiak, S. E. and Cane, M. A.: A model El Niño-Southern Oscillation, Mon. Weather Rev., 115, 2262-2278, 1987.

Zebiak, S. E.: On the 30-60-day oscillation and the prediction of El Niño, J. Climate, 2, 1381-1387, 1989. 